\title{
REMOVAL OF CUMULUS CELLS FROM MAMMALIAN OOCYTES IN A MICROFLUIDIC SYSTEM
}

\author{
H. C. Zeringue and D. J. Beebe \\ Beckman Institute for Advanced Science and Technology, University of Illinois at Urbana-Champaign, \\ Urbana, IL 61801 \\ Department of Biomedical Engineering, University of Wisconsin-Madison, Madison, WI 53706 \\ M. B. Wheeler \\ Department of Animal Science, University of Illinois at Urbana-Champaign, Urbana, IL 61801
}

\begin{abstract}
We have designed, fabricated, and tested a micro fluidic system for the removal of cumulus cells from unfertilized mammalian oocytes. Building devices in polydimethylsiloxane (PDMS) with channel dimensions on the same order as that of the oocyte-cumulus complex $(\sim 400 \mu \mathrm{m})$ allows for the tracking and fluidic manipulation of individual complexes. The system uses pressure driven flows to achieve positioning and movement of the oocyte complex leading to the complete separation and removal of the cumulus cells. A series of narrowed regions are used to "condition" the cumulus. The complex is then brought to ports in the channel that mechanically remove the cumulus. This technique could be combined with other assisted reproductive processes that are amenable to automation in microfluidic systems thereby reducing labor costs and potentially improving in vitro production efficiencies.
\end{abstract}

\section{INTRODUCTION}

The use of in vitro embryo manipulation is expanding as new applications emerge which include: increasing genetic improvement of livestock breeding herds; producing transgenic animals that generate pharmaceutical products in their milk; producing chimeric animals for cancer and genetic disease research; and enabling infertile couples to become pregnant. One of the key steps in many of these processes is in vitro fertilization (IVF). The oocyte (unfertilized egg) is surrounded by the granulosa cell mass, called the cumulus oophorus. The cumulus mass has a consistency similar to honey and is approximately spherical with a diameter between 200 and $500 \mu \mathrm{m}$ for most mammalian species (including mice, swine, cattle, and humans). The cumulus cells are a barrier to sperm penetration. For this reason, the cumulus mass is often removed prior to IVF and embryo maturation [1]. A typical procedure for cumulus removal includes high-speed vortexing of embryos in a centrifuge tube. This causes high stresses on the oocyte complex and is done on large numbers of oocytes simultancously. Oocyte to oocyte contact facilitates the cumulus removal $[2,3]$.

The ability to handle and track individual embryos is very important. Eggs removed from the donors are typically handled in batches to save costs. However, it is advantageous in some cases to keep track of each egg individually [4] from the time it is removed from the donor, through the transfer to the recipient, until the birth of a healthy offspring.
Microfluidic devices have been used for cell manipulation [5-7]. Glasgow et al [8] have shown precise manipulation and retention of single embryos in microfluidic systems. The size scale of the channel environment and laminar flow characteristics of microfluidic systems make them well suited to the automation of embryological procedures. Being able to position and retain an embryo during testing or culture allows the individual identity of each embryo to be maintained. These advantages are employed in the present research to achieve microfluidic cumulus removal.

\section{DEVICE DESIGN, FABRICATION, AND METHODS}

The microfluidic device for removing the cumulus mass (Figure 1) has the following key features: 1) non-toxicity; 2) properly sized cross-sectional geometry; 3) simple loading scheme; 4) "conditioning regions" and ports for mechanical stripping of the cumulus; and 5) connections that allow precise control of the fluid.

The device reported here (Figure 1) is fabricated with glass and PDMS using recently developed techniques[9]. Chan et al [10] have shown that many typical microfabrication materials,

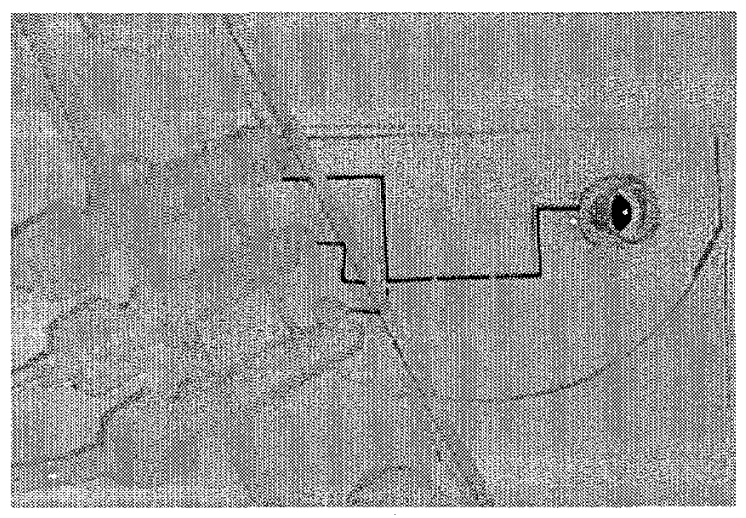

Figure 1: Picture of a device with the inlet well (right) and outlet connections (lefi).

including PDMS, are not toxic to embryos. The main channels in the devices (Figures 1 and 2) are sized to allow a single oocytecumulus complex to travel freely. These channels are $400 \mu \mathrm{m}$ deep by $500 \mu \mathrm{m}$ wide.

A polypropylene well (Figure 1) is bonded to the loading port to provide a larger fluid reservoir at the inlet. An acrylic syringe connection module (Figure 3 ) is clamped over the exit ports so that standard syringes or other fittings can be connected to the device. Syringes enable manual pressure control, or a syringe 
pump can serve as a precise flow controller. Similar to previous glass/silicon devices[8], the PDMS devices allow for embryo positioning throughout a channel network and parking of the oocyte at desired locations during testing. In addition, the PDMS devices provide complete optical access (important for embryo analysis), rapid prototyping, and easy integration with future analysis sensors.

Loading oocytes in the device is simplified through the use of a funnel-shaped inlet well (Figure 2b). The funnel shape is molded at the entrance to the channel with the tip of the funnel connected to the head of the channel. This configuration allows an oocyte complex to bc easily inserted

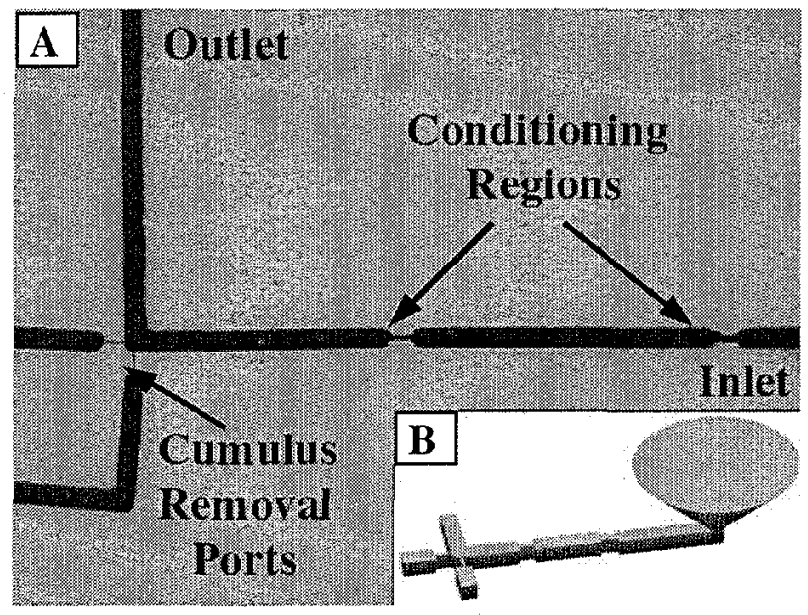

Figure 2: (a) Picture of an actual device with parts labeled. (b) An isometric view of the channel design showing the funnel shaped inlet well.

in the wide funnel opening using standard embryo handling methods. Oocytes will typically sink to the funnel bottom. The sloped walls guide the complex into the channel entrance at the bottom of the funnel. This method of loading simplifies the handling procedures because it does not require precise lateral positioning .

To manipulate the cumulus cells into a configuration that allows for complete cumulus removal, the complex is passed through two narrow channel regions, termed "conditioning regions" (Figure 2a). These narrowed regions

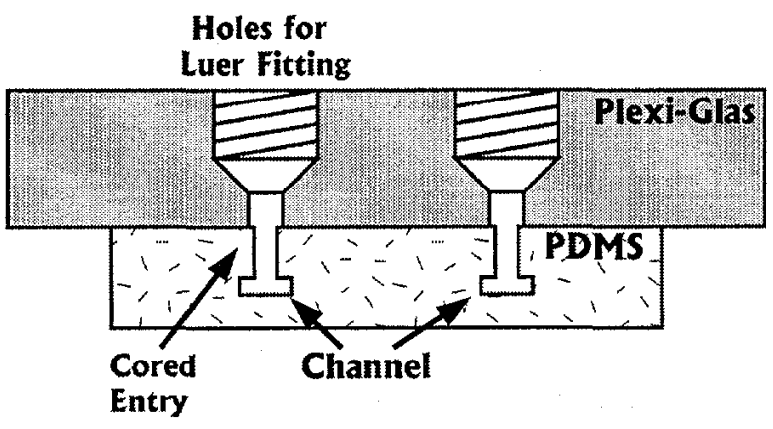

Figure 3: Cross-sectional schematic view of the connection module on the PDMS device. force the cumulus into two main clumps at the front and back of the oocyte (Figure 4a). The two conditioning regions (Figure 2a) are 200 and $150 \mu \mathrm{m}$ wide, respectively.

From the conditioning regions, the oocyte complex flows to the removal ports (Figure $4 \mathrm{~b}$ ). The removal ports are two thin
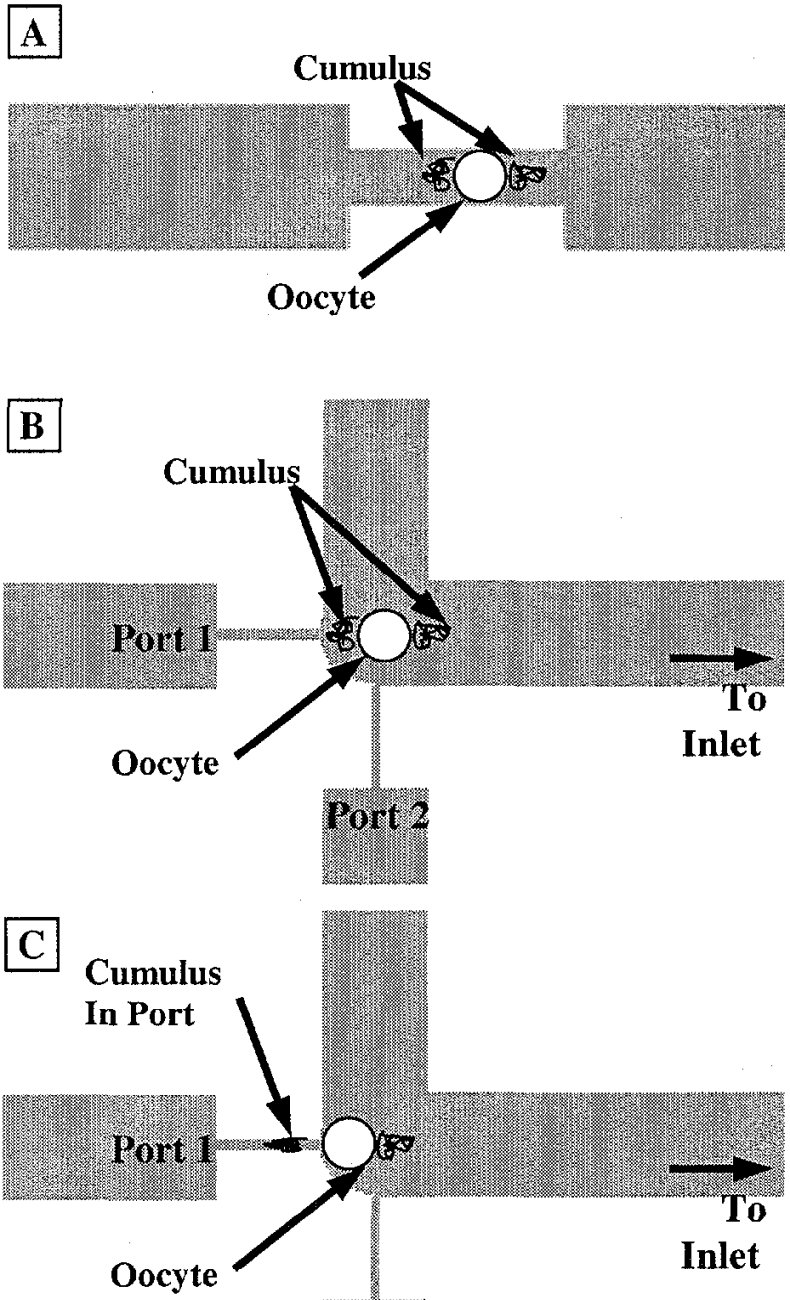

Port 2

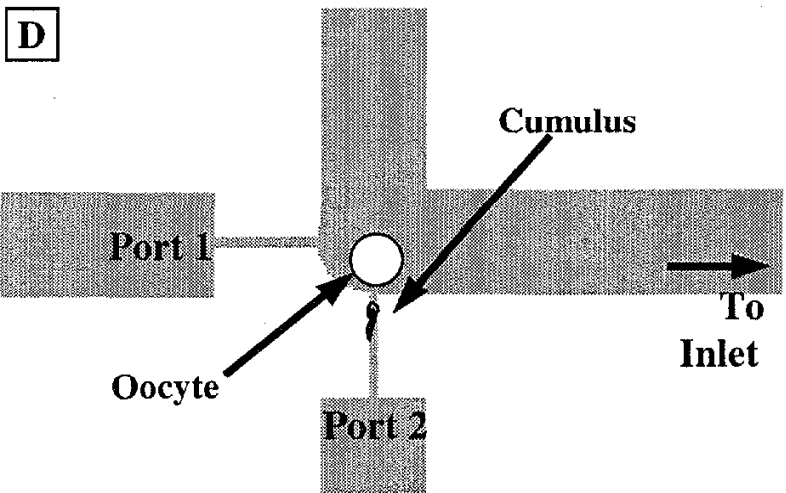

Figure 4: Diagram of the cumulus removal process. The oocyte-cumulus complex is brought to port 1 after passing through the conditioning regions (a). The first bundle of cumulus is removed at port 1 (b). The complex is moved to port 2 where the second bundle of cumulus is removed leaving the clean oocyte (c). 

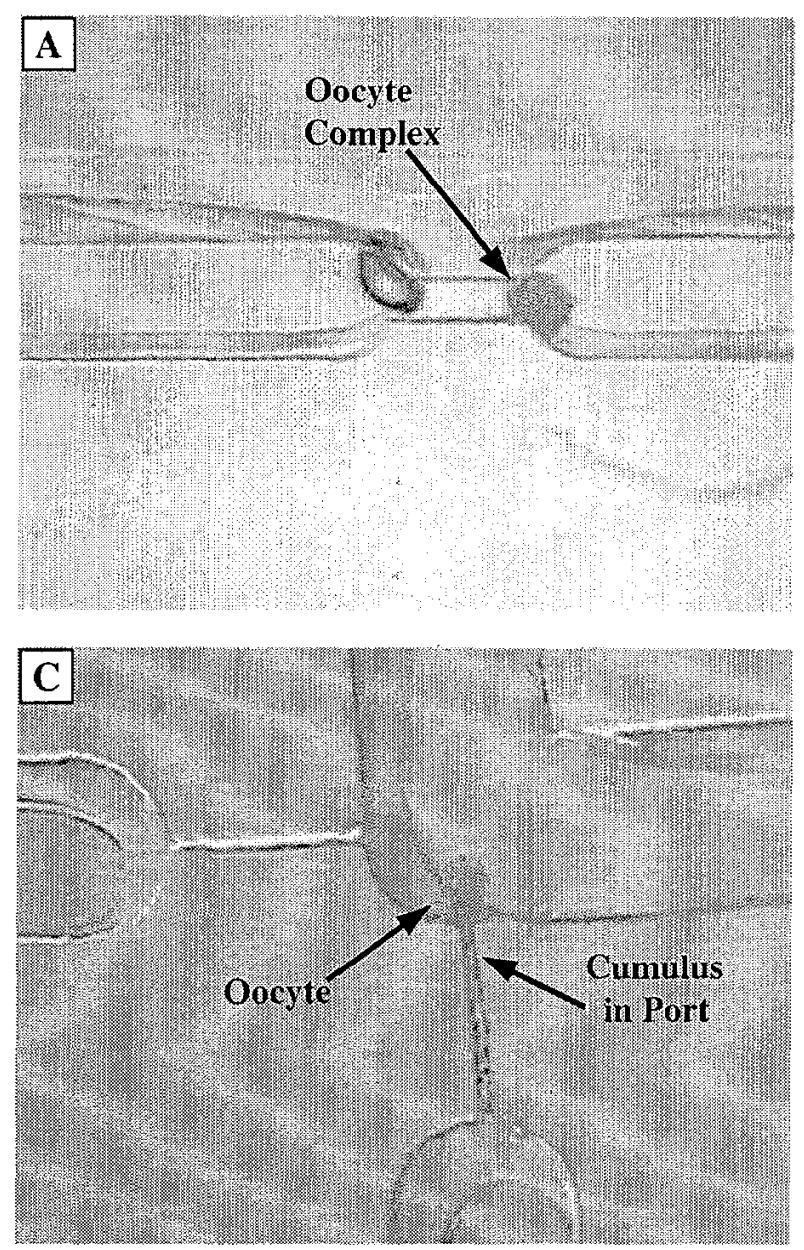

Figure 5: The oocyte-cumulus complex entering the first conditioning region (a). The same complex at the cumulus removal port $1(b)$ and again at port $2(c)$. The clean oocyte in the elbow region (d). The variations of the channel walls are due to undercutting of the epon master during device fabrication. This undercut was transferred to the PDMS during the molding process.

channels placed 90 degrees from one another at a bend in the channel (Figures 2 and 4). The ports allow the cumulus to enter while being too small for the oocyte (Figures $4 c$ and 4d). Using fluid flow, the cumulus can be stripped from the oocyte and carried out of the embryo environment via the port (Figure 4c). The ports are placed at 90 degrees to allow controlled removal of the main cumulus masses. After the first mass has been removed, the oocyte is pulled fluidically to the second port, where it aligns to facilitate removal of the second mass (Figure $4 d$ ).

\section{RESULTS AND DISCUSSION}

Cumulus removal from bovine oocytes has been achieved in a microfluidic system. The cumulus removal process was performed under a stereomicroscope. Initial testing was done using presumptive zygotes from matured bovine oocytes with the cumulus cells intact (oocyte-cumulus complex). Using syringes connected by tubing to the outlet ports (Figure 1), the network was flushed with M16 (Eagel's modified media) then flushed with fresh M16 to remove
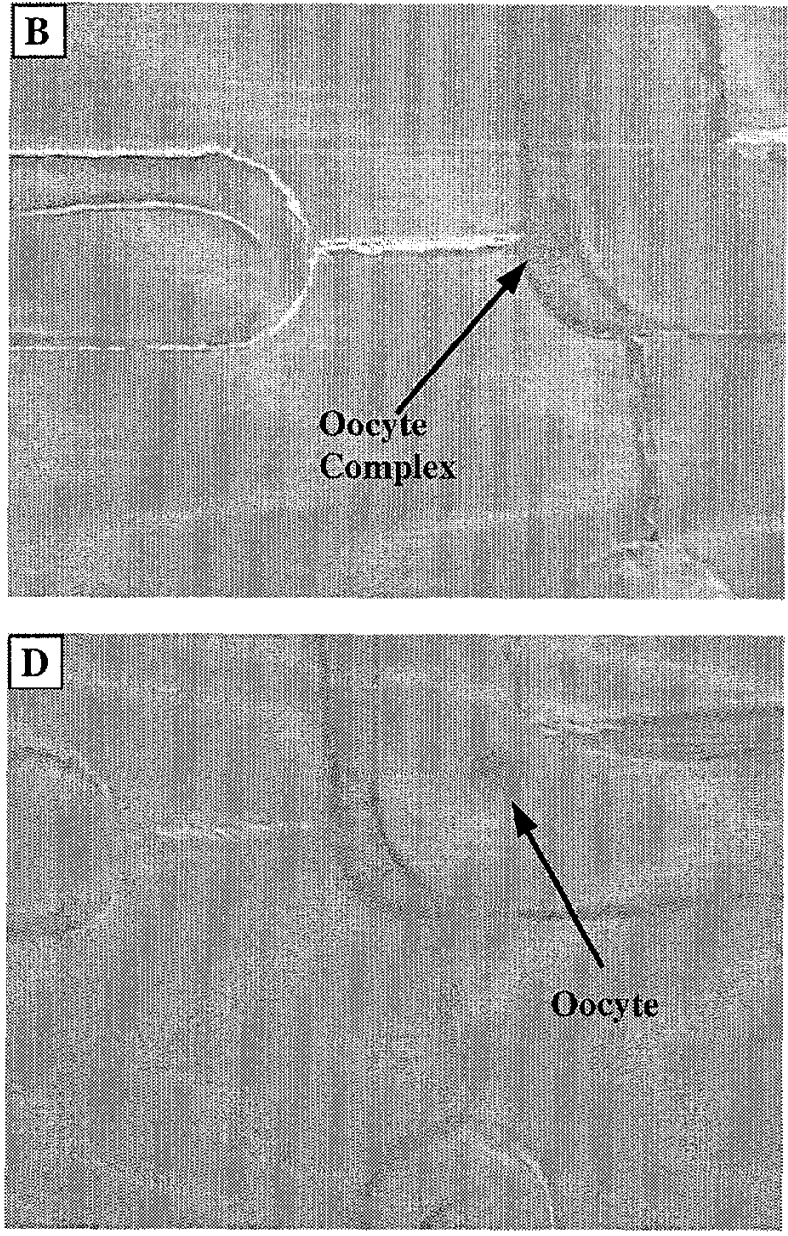

bubbles from the channels. Using fine-bore glass pipettes (a standard embryology tool) an oocyte-cumulus complex was placed in the entrance funnel and allowed to sink to the bottom (20-30 $\mathrm{sec})$. The fluid was then drawn into the channel using the syringes connected to the outlet ports. This caused the complex to move down the channel through the two conditioning regions (Figure 5a). When the complex cleared the second conditioning region, the syringes were adjusted to direct flow to outlet port 1 . The fluid carried the oocyte such that the cumulus entered removal port 1 ahead of the oocyte (Figure 5b). By drawing on the syringe, flow was increased until the cumulus in the port was removed. The exit flow was then switched to exit from outlet port 2 . The complex aligned itself such that the remaining mass of cumulus entered the port (Figure 5c). Again, flow was increased until the cumulus was removed. This left a stripped oocyte at the elbow region (Figure 5d). By reversing the flow in the device, the oocyte was transported back through the channel and back into the funnel entrance region. It could then be easily removed via pipetting techniques.

The cumulus mass normally surrounds the oocyte in an arbitrary manner. Initial testing revealed that forcing the complex through a narrow channel actively grouped the cumulus mass in a non-uniform pattern. The conditioning regions act to reconfigure the cumulus cells into two main bundles. As the oocyte complex passes through the conditioning regions that are the same size as the oocyte, the cumulus cells are forced to the front and rear. The stiffness of the oocyte allows it to hold it's shape while passing through these regions while the cumulus will conform to the 
surrounding spatial restrictions (Figure 4a). The bundles of cumulus cells can then be easily removed via fluid flow. When the complex is brought to a region where the channel is too small for the oocyte to fit, the cumulus will move into this region and can then be sheared from the oocyte using fluid flow (Figures $4 c$ and $4 d$ ). The device presented here utilizes this phenomenon to achieve controlled removal of the cumulus mass.

\section{CONCLUSION}

In this paper, we present a microfluidic platform for cumulus removal from mammalian oocytes. Using pressure driven flow in micro channels allows for the removal of the cumulus from single oocytes. Precise control of oocyte placement and flow rates allow for controlled pressures yielding predictable cumulus removal. This work illustrates the potential impact micro technologies will have on the embryology industry. The ability to precisely control embryo position and mechanically manipulate the local environment are also important enablers of basic developmental biology studies. Future work will include fertilization. Viability tests to ensure that embryo health is not adversely affected by these procedures are planned.

\section{ACKNOWLEDGEMEN'TS}

Rick Munson and Jack Rutledge, Department of $\Lambda$ nimal Sciences, University of Wisconsin-Madison (Madison, WI 5.3706) supplied hovine oocyte-cumulus complexes. Funding for this project was provided by the Illinois Council on Food and Agricultural Research and Univeristy of Wisconsin Madison. Glennys Mensing and Ian Glasgow were very helpful in the proofreading of this document.

\section{REFERENCES}

1. Bavister, B.D., "Analysis of Culture Media for In Vitro Fertilization and Criteria for Success", in Fertilization and Embryonic Development In Vitro, J. L. Mastroianni and J.D. Biggers, Editors. 1981, Plenum Press: New York. p. 41-60.

2. Rutledge, J, and R. Munson, Pcrsonal Communication, Department of Animal Sciences, University of WisconsinMadison. February 2000.

3. Wang, L., and David J. Beebe, Allen R. Williams, Kim D. Easley, "Electrothermal Branding for Embryo Labeling",. IEEE Transactions on Biomedical Engineering, 1997.

4. Li, P.C.H. and D.J. Harrison, "Transport, Manipulation, and Reaction of Biological Cells On-Chip Using Electrokinetic Effects", Analytical Chemistry, 1997. 69(8): p. 1564-1568.

5. Austin, R.H., R.H. Carlson, S.S. Chan, J. Knight, S. Turner, and H. Craighead. "Micro and Nanopore Structures for Biological Applications", in 1997 International Conference on Solid-State Sensors and Actuators. 1997. Chicago, IL.

6. Glasgow, I., H.C. Zcringue, D.J. Bcebc, S.-J. Choi, J.T. Lyman, and M.B. Wheeler. "Individual Embryo Transport and Retention on a Chip" in $\mu T A S$ '98. 1998. Banff, Canada.

7. Duffy, D., J.C. McDoanld, O.J.A. Schueller, and G. Whitesides, "Rapid Prototyping of Microfluidic Systems in Poly (dimethyl siloxane)", Angewandte Chemie. International edition in English, 1998. 37: p. 550-575.

8. Chan, N.G., J.T. Lyman, S.-J. Choi, H.C. Zeringue, I.K. Glasgow, D.J. Beebe, and M.B. Wheeler, "Development of an Embryo Transport and Analysis System: Material Biocompatability". Theriogenology, 1999. 51(1): p. 234. 